\title{
Dynamics of Network Connectivity in Tactical MANETs
}

\author{
Younes Regragui \\ LAROSERI, Department of Computer Science \\ Chouaib Doukkali University, El Jadida Morocco. \\ Email: regragui.y@ucd.ac.ma
}

\author{
Najem Moussa \\ LAROSERI, Department of Computer Science \\ Chouaib Doukkali University, El Jadida Morocco. \\ Email: moussa.n@ucd.ac.ma
}

\begin{abstract}
In the future battlefields, communication between commanders and soldiers will be a decisive factor to complete an assigned mission. In such military tactical scenarios, network topology is constrained by the dynamics of dismounted soldiers in the battlefield. In the battlefield area, soldiers may be divided into a number of squads and fire teams with each one having its own mission, especially in some critical situation (e.g., a military response to an enemy attack or a sweep operation of houses). This situation may cause an unpredictable behavior in terms of wireless network topology state, thus increasing the susceptibility of network topology to decomposition in multiple components. This paper presents a Group Mobility Model simulating realistic battlefield behaviors and movement techniques. We also analyze wireless communication between dismounted soldiers and their squad leader deployed in a mobile ad hoc network (MANET) under different packet sending rate and perturbation factor modeled as a standard deviation parameter which may affect soldiers' mobility. A discussion of results follows, using several performance metrics according to network behavior (such as throughput, relaying rate of unrelated packets and path length).
\end{abstract}

Index Terms-Self-Organized Behavior, Mobility Models, Ad hoc Networks, Group mobility, Dismounted soldiers, Battlefield.

\section{INTRODUCTION}

Recently, the army has been interested in developing new skills and using more efficient technological systems for dismounted soldiers which can be adapted to their needs on the battlefield area and incorporates all modern combat equipment such as making soldiers more connected [1] in the battlefield based on modern soldiers' electronic equipment and computer technologies by using mobile wireless ad hoc networks (MANETs) [2], [3].

In the battlefield area, communications network and information sharing are more important for mission execution by dismounted soldiers. The challenges of Military Tactical Networking is related to military-style tactical operations, where dismounted soldiers should interact simultaneously with the environment (battlefield) and with each other so as to complete an assigned mission such as a sweep operation of houses or buildings in a wide area, containing features such as mountains, forests, or rivers. This stressful overcharge of interactions in battlefield area, in conjunction with the geographically variable nature of the battlefield area and the unpredictable behavior in terms of wireless network topology state, thus increasing the susceptibility of network topology to decomposition in multiple components. The network connectivity is worsened due to topology changes driven by wireless links and unpredictable mobility of soldiers. Thus, it is natural that the highly dynamic in nature of dismounted soldiers' mobility on the battlefield has a significant impact on the network topology, eventually resulting in high rate of link failures [4]-[6]. Therefore, the evaluation of the network performance during the execution of a tactical military scenario for such situations in the real world setting is in many cases not feasible since the cost can be too high and it is impossible to test in real world. Therefore, a simulation environment is very attractive for evaluating and studying the impact of soldiers' behaviors on the performance of the network and topology condition during military operations.

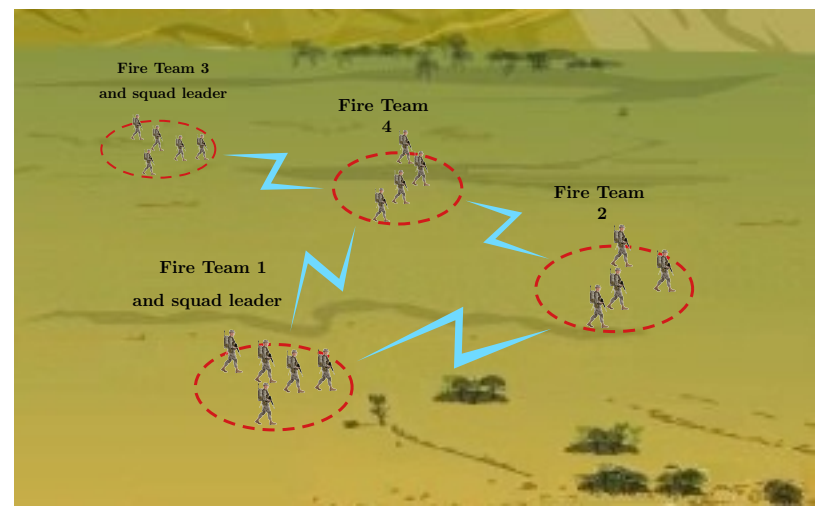

Fig. 1. Illustration of dismounted soldier group in military modern communications infrastructure.

In this paper, we propose a novel group mobility model to simulate the mobility of soldiers by using a wedge formation as basic movement technique as realistically as possible and analyze the effectiveness of wireless communications on the battlefield between dismounted soldiers.

The rest of the paper is organized as follows. The related work is presented in Section 2. Section 3 describes the collective motion approach we have used in our group mobility model. Experimental results are presented in Section 4. Section 5 concludes the paper and discusses the future research. 


\section{RELATED WORK}

In this section, we will review existing Group mobility models and tactical networks in MANET in the literature which make efforts to realistically model the mobility of nodes and scenarios for tactical military applications in MANETs. The Reference Point Group Mobility Model (RPGM) [7] is the most widely known of the group mobility models. Each group has a logical "center" which defines the entire group's motion behavior, including location, speed, direction and acceleration. In [8], the Reference Velocity Group Mobility Model (RVGM) extends the RPGM model by proposing two categories of velocity vectors: group velocity of logical center or the lead point, and the local deviation velocity of each group member. In [9], the authors introduced the Group Force Mobility Model (GFMM) which is similar to RVGM in that the velocity of a group member follows the velocity of its lead point with a small random deviation.

In recent years, several approaches have been interested in MANET for tactical and emergency scenarios. For example, in [10], the authors proposed a mobility model (Hierarchical Group Mobility model) designed for modeling a military operation in order to study the connectivity of a MANET established through wireless communication between the moving objects (ships, vehicles or foot soldiers, and aircraft). In [11], the authors introduced a new group mobility model called the Diamond Group Mobility (DGM), where a group of soldiers is restricted in a diamond region with one dismounted soldier at the center considered as (commander). In [12], the authors proposed a virtual track based group mobility model (VT model) which closely approximates the mobility patterns in military MANET scenarios. In [13], the authors proposed tactical MANET Content Centric Networking (CCN) for emergency wireless ad hoc environments. In [14], the authors described the networking usage requirements for MANET over legacy narrowband tactical waveforms and discussed the common characteristics of legacy tactical radio waveforms and the implications of such characteristics for the MANET implementation.

\section{Characterising AND MODElling Dismounted SOLDIERS' MOBILITY}

This section presents the basic concept of our group mobility model which govern the various aspects of mobility within the group environment. It describes how the movement of dismounted soldiers is modeled on the battlefield based on a set of simple rules in order to make soldiers move collectively. These rules also are able to make each soldier behaves autonomously without a central control. The selforganized behavior of independent agents is inspired by observable characteristics of many categories of social cohesion and animals' aggregation behavior which are found in nature (e.g. ant colonies, birds flock flying and fish swarms, soldiers in the battlefield) carry out their tasks collectively in order to contribute to a common goal [15], [16]. Thus, the mobility of an agent can be governed by three basic rules (i.e. cohesion, separation and alignment).

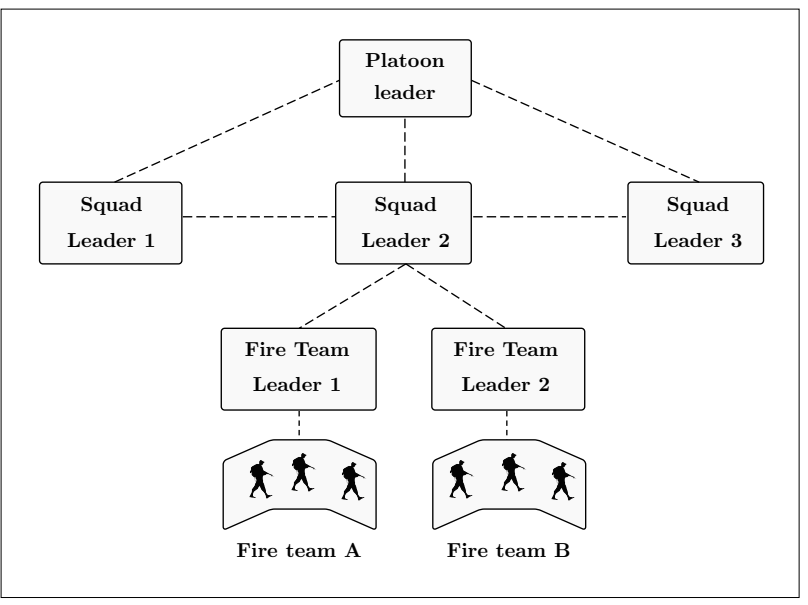

Fig. 2. Organizational structure of a platoon.

We assume that $N$ dismounted soldiers move at a constant speed of $v_{0}$ units per second. Each soldier is characterized by his location $\mathbf{p}_{i}(t)$ and velocity $\mathbf{s}_{i}$ of direction $\mathbf{d}_{i}(t)$ at time $\mathrm{t}$. In each time step $t$, a member $i$ assesses the position and/or orientation of neighbors in its local neighborhood within two non-overlapping behavioral zones (Fig. 5) to determine its desired direction of motion $\mathbf{d}_{i}(t+\Delta t)$ at time $t+d t$. After that, the member $i$ turns towards the direction vector $\mathbf{d}_{i}(t+\Delta t)$ by the turning angle $\alpha_{i} \Delta t$, where

$$
\alpha_{i}=\sigma+\chi
$$

where $\sigma$ is the turning rate, $\chi$ is a random angle taken from a circular-wrapped gaussian distribution, centered on 0 , with standard deviation $\chi$ radians gaussian-distributed angle. The location of the member $i$ at time $t+\Delta t$ is given by:

$$
\mathbf{p}_{i}(t+\Delta t)=\mathbf{p}_{i}(t)+\mathbf{v}_{i}(t+\Delta t) \Delta t \mathbf{s}_{i}
$$

\section{A. Behavioral rules description}

In this section, we take into account the ability of grouping individuals within two independent groups (e.g., the lead team and trail team) and the degree of interaction between them needed to develop dominance relationships. These relationships allow individuals move together as one group forming a rifle squad. Based on the interactions between soldiers and teams, our potential purpose is further to model a squad column known as a movement formation and a movement technique used in battlefield area. In the battlefield area, the squad traveling movement technique is used when enemy contact is not likely. This movement technique offers the best speed and control during maneuver, the trail team follows closely behind the lead fire team. In this paper, we are interested in incorporating both the movement formation and movement technique of a squad by considering a mathematical modeling and a set of simple rules responsible of soldiers' behaviors. 


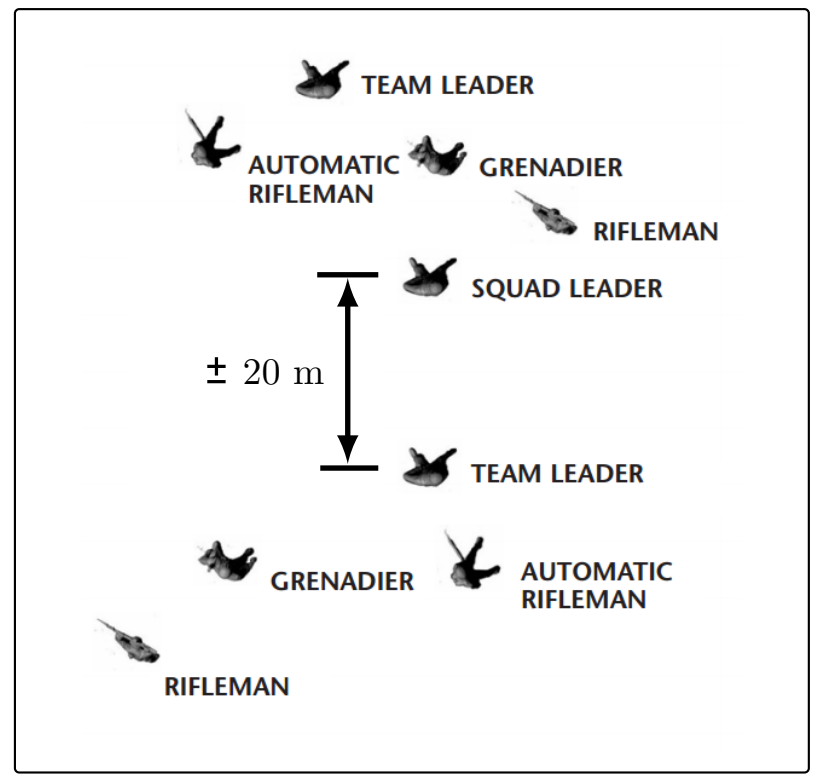

Fig. 3. Squad Traveling.

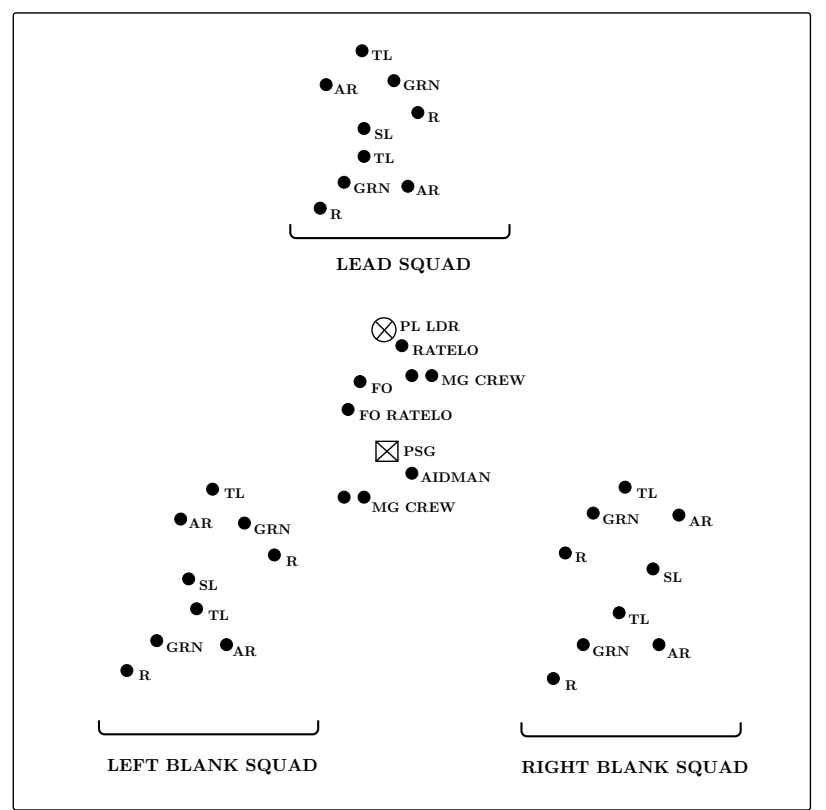

Fig. 4. Illustration of wedge formation which has two squads in the rear that can overwatch or trail the lead squad.

1) Repulsion behavior: Each dismounted soldier $i$ attempts to maintain a minimum distance from neighbors within the repulsion zone (ZoR), modeled as a circle, centered on the dismounted soldier $i$ with radius $R_{1}$. If $n_{r}$ neighbors are present in this zone at time $t$, the direction of repulsion from neighbors is given as follows:

$$
\mathbf{d}_{i}(t+\Delta t)=-\sum_{j \neq i}^{n_{r}} \frac{\mathbf{r}_{i j}}{\left|\mathbf{r}_{i j}\right|}
$$

where $\mathbf{r}_{i j}=\left(\mathbf{p}_{j}-\mathbf{p}_{i}\right) /\left|\left(\mathbf{p}_{j}-\mathbf{p}_{i}\right)\right|$ is the unit vector from the location point of soldier $i$ in the direction of the neighbor $j$.

2) Attraction and orientation behaviors: Each dismounted soldier $i$ attempts to become attracted towards $n_{a}$, and aligned with $n_{o}$ neighbours within both the zone of attraction and orientation ( $\mathrm{ZoA}$ and $\mathrm{ZoO})$, centered on the dismounted soldier $i$ with radius $R_{2}$.

$$
\mathbf{d}_{i}(t+\Delta t)=\sum_{j}^{n_{o}} h_{j} \frac{\mathbf{v}_{j}}{\left|\mathbf{v}_{j}\right|}+\sum_{j \neq i}^{n_{a}} h_{j} \frac{\mathbf{r}_{i j}}{\left|\mathbf{r}_{i j}\right|}
$$

where $\mathbf{v}_{j}$ is a unit direction vector of soldier $j$. In our scenario, $h_{j}$ is always equals to 1 except of squad leader, where $h_{j}=2$ so as to give him a higher priority [17].

Here $\mathbf{d}_{i}(t+\Delta t)$ is converted to the corresponding unit vector $\hat{\mathbf{d}}(t+\Delta t)=\mathbf{d}_{i}(t+\Delta t) /\left|\mathbf{d}_{i}(t+\Delta t)\right|$.

To incorporate the influence of informed group members, a proportion of the individuals $p$ are given information about a preferred direction (simulated as a unit vector $\mathrm{g}$ ) representing the destination to a known resource or enemy location [18]. However, in our considered scenario $(p=1)$. Then, the informed individuals balance the influence of their preferred direction and their social interactions with weighting term $\omega$, as follows:

$$
\mathbf{d}_{i}^{\prime}(t+\Delta t)=\frac{\hat{\mathbf{d}}_{i}(t+\Delta t)+\omega g}{\left|\hat{\mathbf{d}}_{i}(t+\Delta t)+\omega g\right|}
$$

where $\omega$ is weighting term used by the members of lead team, whereas the members of trail team use $\omega^{\prime}$ as weighting term towards the lead team (e.g. the destination of trail team is the lead team). The destination position is chosen randomly within the simulation area.

After a detailed analysis of the variation of $\omega^{\prime}$ as a function of $\omega$ (see Fig. 6), we found that $\omega^{\prime}$ is given as follows:

$$
\omega^{\prime}= \begin{cases}\frac{d}{d_{0}}\left[\exp \left(d-d_{0}\right)+\ln \left(\frac{\omega}{4}\right)\right] & \omega<1, \\ \frac{d}{d_{0}}\left[\exp \left(d-d_{0}\right)+\omega\right] & \text { Otherwise. }\end{cases}
$$

where Fig. 6 shows the variation of distance between trail team and lead team based on definition of $\omega^{\prime}$ in Eq.6. The results show that the distance $\mathrm{d}$ keep almost equals to the limit distance $d_{0}(\simeq 20 \mathrm{~m})$.

After the above process has been performed for every member, all members can turn through an angle of, at most, $\alpha_{i} \Delta t$ radians towards the desired direction $\hat{\mathbf{d}}_{i}(t+\Delta t)$ at time $(t+\Delta t)$ by the turning rate $\sigma$;if the angle between $v_{i}(t)$ and $\hat{\mathbf{d}}_{i}(t+\Delta t)$ is less than $\alpha_{i} \Delta t$, they achieve alignment with their desired vector, $v_{i}(t+\Delta t)=\hat{\mathbf{d}}_{i}(t+\Delta t)$, otherwise they turn $\alpha_{i} \Delta t$ towards it. We apply this process at each time step $\Delta t$, where each member is able to independently perform behavioural responses to maintain interactions with other members in its neighborhood. 


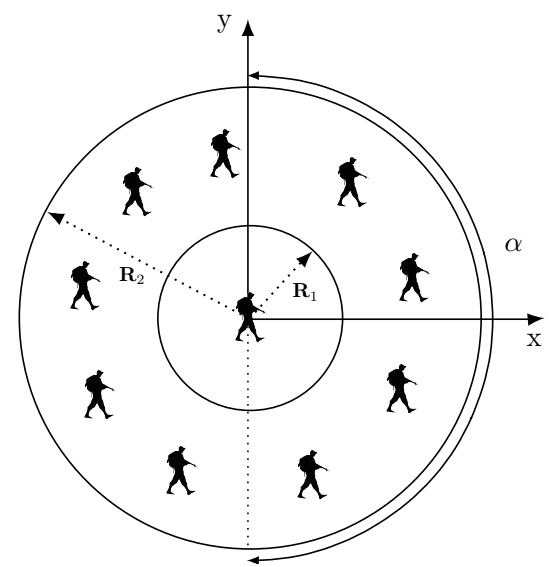

Fig. 5. Representation of a member in the model centered at the origin: $R_{1}=$ zone of repulsion, $R_{2}=$ zone of orientation and attraction, and $\alpha$ is the field of perception ahead of the member.

TABLE I. Mobility model parameters used in the simulation.

\begin{tabular}{|l||c||c|}
\hline \multicolumn{1}{|c|}{ Parameter } & Symbol & Value \\
\hline Number of soldiers & $\mathrm{N}$ & 9 \\
Zone of repulsion & $Z_{o R}$ & $10 \mathrm{~m}$ \\
Zone of attraction & $Z_{o A}$ & $50 \mathrm{~m}$ \\
Zone of orientation & $Z o O$ & $50 \mathrm{~m}$ \\
Number of groups & - & 1 \\
Number of teams & - & 2 \\
Turning rate & $\sigma$ & $2 \mathrm{rad}$ \\
Initial velocity of nodes & $v_{0}$ & $1 \mathrm{~m} / \mathrm{s})$ \\
Packet sending rate & $\lambda$ & $0.2(\mathrm{~s})$ \\
Time step & $d t$ & {$[0,2] \mathrm{rad}$} \\
Standard deviation & $\chi$ & 5 and \\
\hline
\end{tabular}

\section{B. Network Model}

Our group mobility model has been implemented using Java and is able to generate mobility traces. To evaluate our model in terms of network performance, we perform NS-2 simulations to observe and analyze the impact of soldiers' dynamics on reliability of communications using the mobility traces of soldiers in the battlefield area generated from our Java simulator. The squad leader can exchange communications with their neighbors in the squad.

Our network contained $\mathrm{N}$ mobile nodes/soldiers deployed close to each other in a simulation area of $5000 \times 5000 \mathrm{~m}^{2}$. At each time step $d t$. The soldiers/nodes are able to exchange data packets based on AODV routing protocol with a data generation rate $(\lambda)$.

\section{Simulation Results}

In this paper, to study the impact of soldiers' dynamic in the battlefield area on the performance of the network, we evaluated the network performance under different packet sending rate $\lambda$ and perturbation levels $\chi$ (standard deviation). The perturbation of the soldiers' mobility is expected to simulate several effective factors in the mobility environment such as obstacles, crossing forests, and rivers.
TABLE II. Network model parameters used in the simulation.

\begin{tabular}{|l|l|}
\hline \multicolumn{1}{|c|}{ Parameter } & \multicolumn{1}{c|}{ Value } \\
\hline Propagation model & Propagation/TwoRayGround \\
Network area & $5000 \mathrm{~m} \times 5000 \mathrm{~m}$ \\
Packet generator & $\mathrm{CBR}$ \\
Transmission range & $60 \mathrm{~m}$ \\
Simulation time & $1000 \mathrm{~s}$ \\
Queue size & 64 packets \\
Transport protocol & UDP \\
Routing Protocol & AODV \\
CBR packet size & 1000 bytes \\
\hline
\end{tabular}

From Fig. 8, it is shown that the average throughput decreases significantly as a function of the standard deviation. Moreover, when the standard deviation becomes higher, the throughput highly decreases, especially in the range [1.8,2]. We can explain that by the fact that the increase in the standard deviation can hardly cause a topology change due mainly to perturbation of the collective motion of dismounted soldiers allowing soldiers to move with a low accuracy. As a consequence, an arbitrary partition of the network topology occurs when the standard deviation reaches a higher level.

Fig. 9 shows the effect of increasing the standard deviation on relaying rate of packets by intermediate nodes. It is

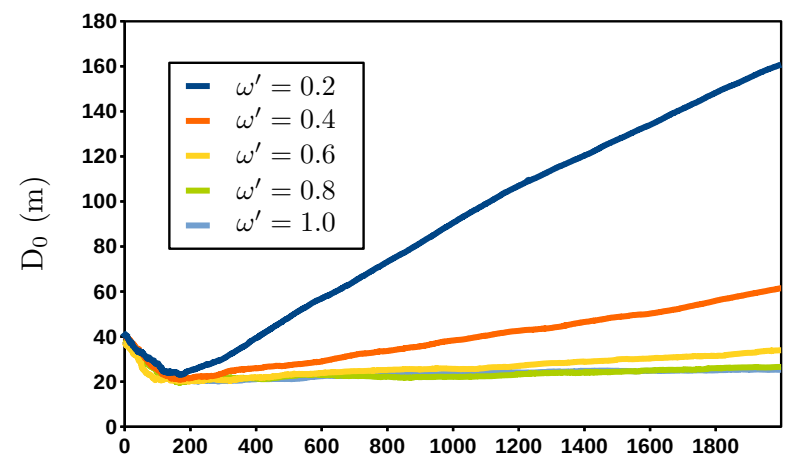

(a)

Time (s)

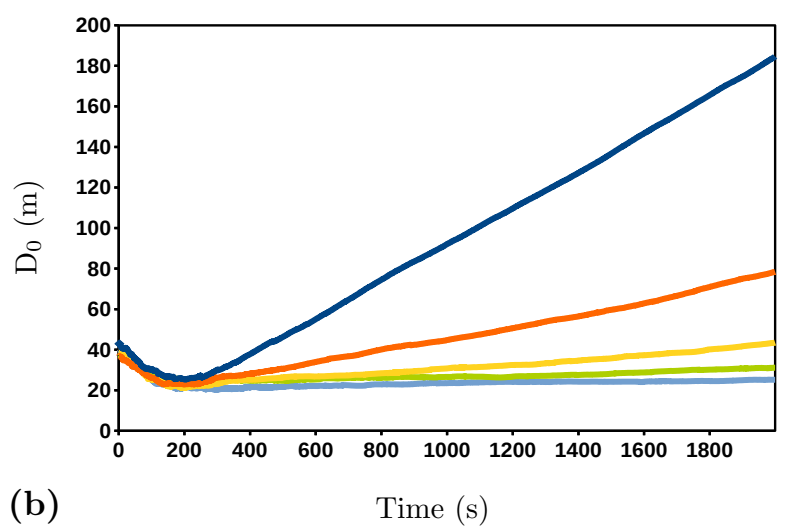

Fig. 6. Effect of increasing the weighting $\omega^{\prime}$ on the distance between trail team and lead team in the case of $\omega=0.5$ (a) and with $\omega=1$ (b) respectively. 


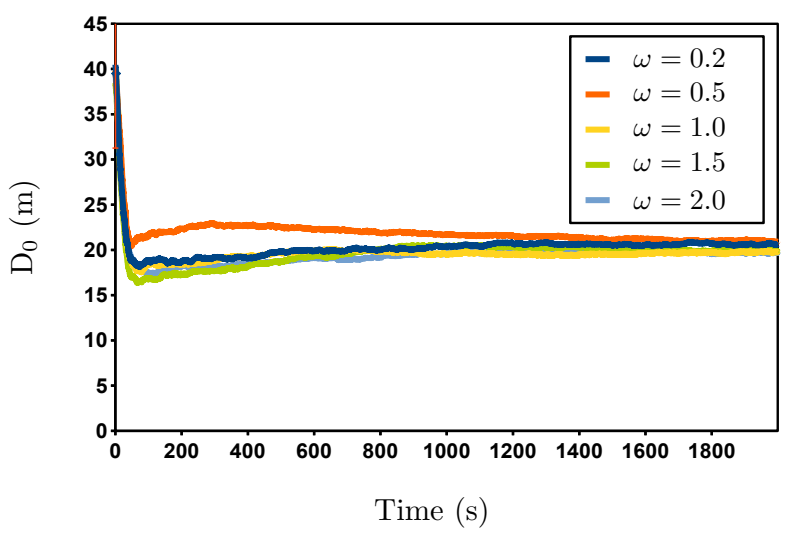

Fig. 7. Effect of increasing the weighting $\omega$ on the distance between trail team and lead team.

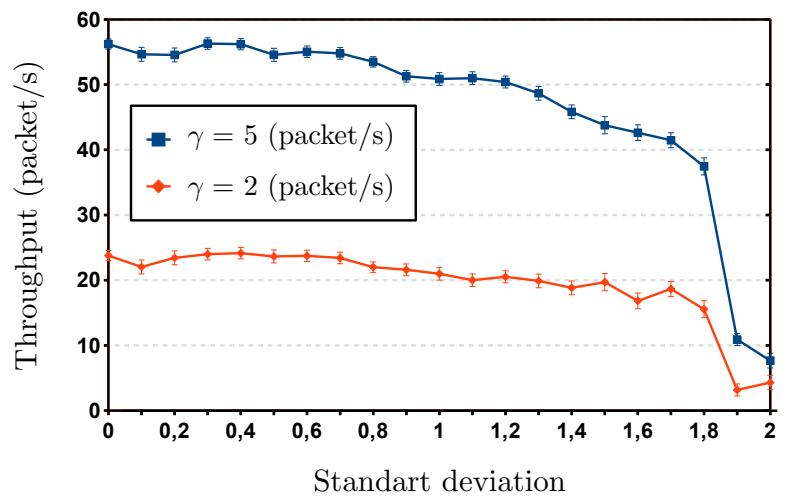

Fig. 8. Effect of increasing the standard deviation on the network throughput.

observed that the relaying rate of packets is also affected by the increase in the standard deviation. This illustrates that the nodes encounter a difficulty to find stable paths for relaying unrelated packets as the standard deviation increases. This is due mainly to bad condition of network topology which results from an increase in the link failures when the distance between two soldiers becomes higher than the transmission range.

Fig. 10 shows that the path length increases as the standard deviation increases until reaching a maximum length at 0.5 . But, it starts decreasing as the standard deviation continues increasing. We can explain that by the fact that the network topology is elongated when the standard deviation is $(<=0.5)$. However, when the standard deviation is increased enough $(>0.5)$, the network is partitioned info small clusters and isolated nodes. This is sufficient to explain why the path length is decreased: the source node cannot establish a path with the destination node if there are sufficient nodes which can participate as relay nodes in this path.

\section{CONCLUSIONS}

In this paper, we have presented a battlefield collective motion model for dismounted soldier squad, including the interaction between tail and lead teams respectively, based on a detailed modeling and analysis of the dynamic of both teams in

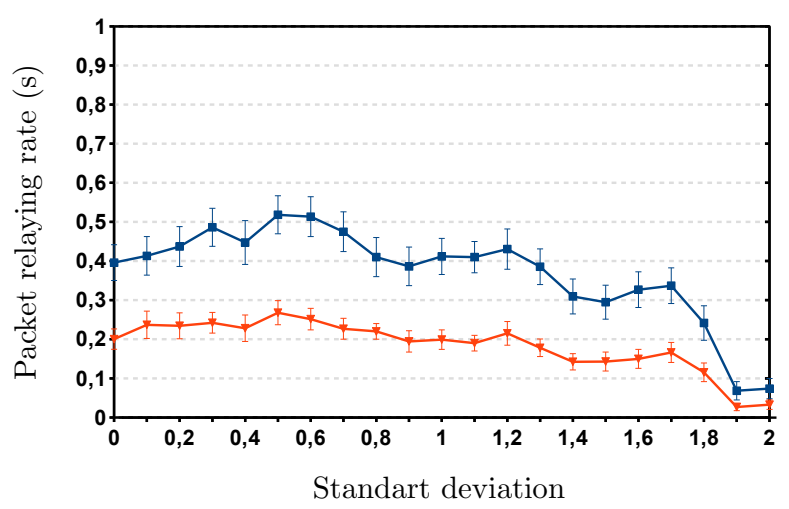

Fig. 9. Effect of increasing the standard deviation on the relaying rate of unrelated packets per node.

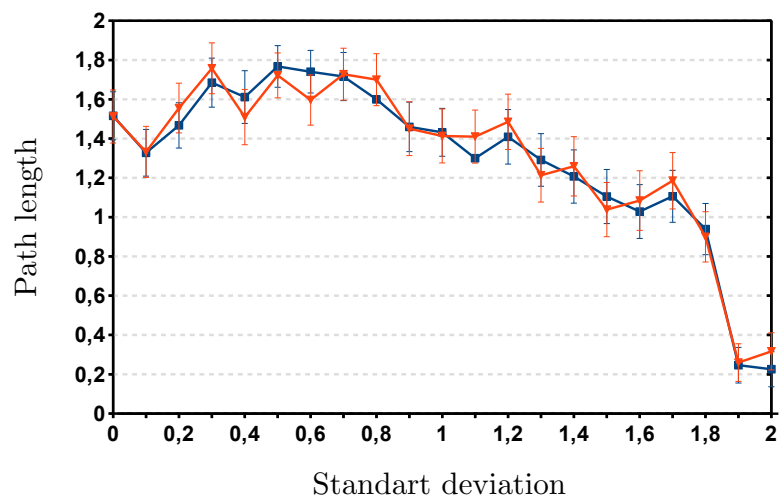

Fig. 10. Effect of increasing the standard deviation on the path length.

the battlefield area. We considered a wedge formation as basic movement technique as realistically as possible. Moreover, we analyzed extensively the considered network scenario under different packet sending rate and perturbation levels of the collective motion of dismounted soldiers.

The simulation results show that the network performance is more sensitive to the dynamic of soldiers in the simulation area. Moreover, the standard deviation has a much higher impact on the throughput, relaying rate of unrelated packets and path length. Moreover, a significant perturbation of network topology is noticed when increasing the standard deviation. This reflects a worse case of the network and its limitation for assuring reliable communications under a high dynamic of soldiers in the battlefield. In our future work, we plan to extend our research to widely adopt a tactical network with several squads, where dismounted soldiers are organized into a platoon and following different tactical formations depending on the current situation on the battlefield area.

\section{REFERENCES}

[1] S. L. Cotton, W. G. Scanlon, E. Skafidas, B. K. Madahar et al., "Millimeter-wave stealth radio for special operations forces," Microwave Journal, vol. 53, no. 8, p. 6, 2010.

[2] N. R. Council, Energy-Efficient Technologies for the Dismounted Soldier. The National Academies Press, 
1997. [Online]. Available: http://www.nap.edu/catalog/5905/ energy-efficient-technologies-for-the-dismounted-soldier

[3] T. J. Thomas, "The defence and national security capability reporter," vol. Vol 28 Nos 04/05 of 2009, no. 48, pp. 1-48, 2009.

[4] Y. Regragui and N. Moussa, "Agent-based system simulation of wireless battlefield networks," Computers \& Electrical Engineering, vol. 56, pp. 313-333, 2016.

[5] A. Zadin and T. Fevens, "Maintaining path stability with node failure in mobile ad hoc networks," Procedia Computer Science, vol. 19, pp. 1068-1073, 2013.

[6] R. S. Abujassar, "Mitigation fault of node mobility for the manet networks by constructing a backup path with loop free: enhance the recovery mechanism for pro-active manet protocol," Wireless Networks, vol. 22, no. 1, pp. 119-133, 2016.

[7] X. Hong, M. Gerla, G. Pei, and C.-C. Chiang, "A group mobility model for ad hoc wireless networks," in Proceedings of the 2nd ACM international workshop on Modeling, analysis and simulation of wireless and mobile systems. ACM, 1999, pp. 53-60.

[8] K. H. Wang and B. Li, "Group mobility and partition prediction in wireless ad-hoc networks," in Communications, 2002. ICC 2002. IEEE International Conference on, vol. 2. IEEE, 2002, pp. 1017-1021.

[9] S. A. Williams and D. Huang, "Group force mobility model and its obstacle avoidance capability," Acta Astronautica, vol. 65, no. 7, pp. 949-957, 2009.

[10] A. Fongen, M. Gjellerud, and E. Winjum, "A military mobility model for manet research," Parallel and Distributed Computing and Networks (PDCN 2009), February, vol. 16, p. 18, 2009.

[11] L. Ning, G. Yan, D. Chao, and W. Jinlong, "Diamond group mobility model for ad hoc network in military," in Signal Processing, 2008. ICSP 2008. 9th International Conference on. IEEE, 2008, pp. 2754-2756.

[12] B. Zhou, K. Xu, and M. Gerla, "Group and swarm mobility models for ad hoc network scenarios using virtual tracks," in Military Communications Conference, 2004. MILCOM 2004. 2004 IEEE, vol. 1. IEEE, 2004, pp. 289-294

[13] S. Y. Oh, D. Lau, and M. Gerla, "Content centric networking in tactical and emergency manets," in Wireless Days (WD), 2010 IFIP. IEEE, 2010, pp. $1-5$.

[14] H. Wang, B. Crilly, W. Zhao, C. Autry, and S. Swank, "Implementing mobile ad hoc networking (manet) over legacy tactical radio links," in Military Communications Conference, 2007. MILCOM 2007. IEEE. IEEE, 2007, pp. 1-7.

[15] C. W. Reynolds, "Flocks, herds and schools: A distributed behavioral model," ACM Siggraph Computer Graphics, vol. 21, no. 4, pp. 25-34, 1987.

[16] I. D. Couzin, J. Krause, R. James, G. D. Ruxton, and N. R. Franks, "Collective memory and spatial sorting in animal groups," Journal of theoretical biology, vol. 218, no. 1, pp. 1-11, 2002.

[17] R. Freeman and D. Biro, "Modelling group navigation: dominance and democracy in homing pigeons," The journal of navigation, vol. 62, no. 1 , pp. 33-40, 2009.

[18] I. D. Couzin, J. Krause, N. R. Franks, and S. A. Levin, "Effective leadership and decision-making in animal groups on the move," Nature, vol. 433, no. 7025, pp. 513-516, 2005. 